\title{
corrigendum
}

\section{2 yellow is the colour of longing}

\author{
K.R. Meera
}

Correction to: Feminist Review (2009) 91, 180-185.

doi: $10.1057 /$ fr. 2008.44

We regret that the translator credit for the above short story, which appeared in the previous issue was omitted inadvertently.

The translator of the Malayalam short story 'Yellow is the colour of longing' by K.R. Meera was J. Devika, Associate Professor, Centre for Development Studies, Thiruvananthapuram, Kerala, India.

doi: $10.1057 /$ fr. 2009.19 